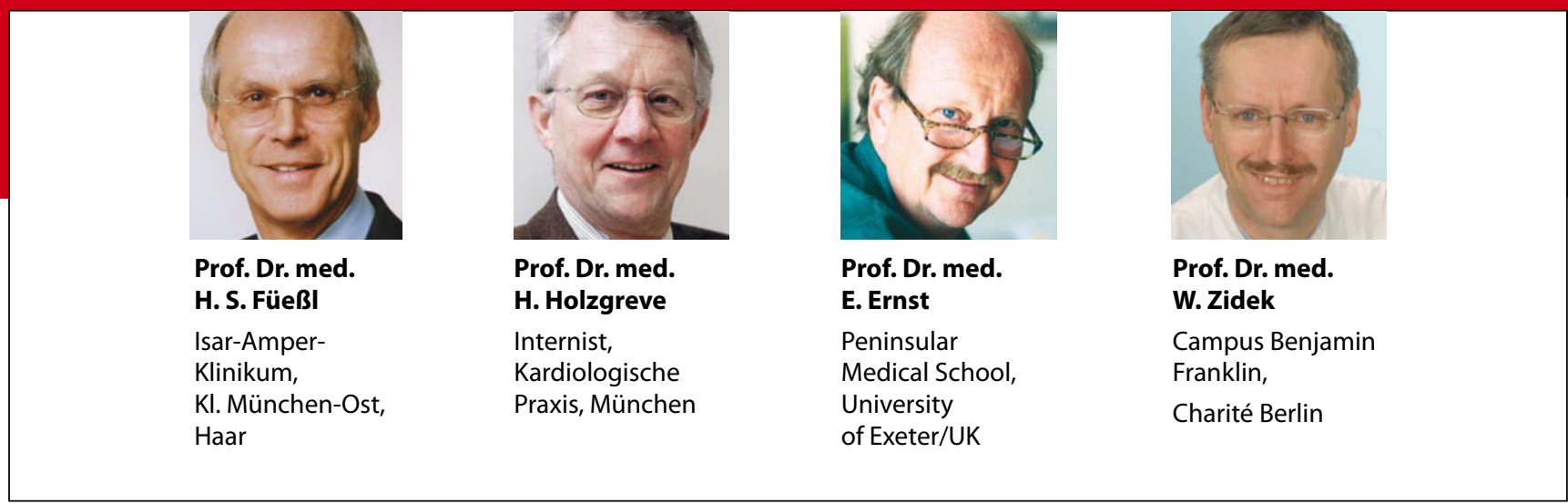

\title{
Weiterer Sargnagel für die Vitamin-E-Supplementation
}

\section{Die diätetische Supplementation der Nahrung mit Vitamin E erhöht bei gesunden Männern signifikant das Risiko für ein Prostatakarzinom.}

- Der Höhenflug des Vitamin E begann vor etwa 20 Jahren, als man aus Beobachtungsstudien und In-vitro-Versuchen weit reichende Schlüsse auf das umfangreiche therapeutische und präventive Potenzial dieses Vitamins zog. Von der Prävention der Arteriosklerose, der Tumorprophylaxe bis hin zum AntiAgeing, Vitamin E galt als Zaubermittel und wurde in der alternativen Medizin mit Begeisterung verordnet.

Später durchgeführte Studien haben den Wert von Vitamin E für all diese Indikationen Schritt um Schritt widerlegt. Nun liegt die Langzeitauswertung der Selen-und-Vitamin-E-Krebspräventions-Studie (Select) vor, bei der über 35500 gesunde Männer in 427 Zentren der USA, Kanadas und Puerto Ricos zwischen 2001 und 2004 in vier Studienarme randomisiert wurden. Jeweils 8700 Männer erhielten $200 \mu \mathrm{g} /$ Tag Selen, 400 IU/Tag Vitamin E, beide Substanzen oder Placebo. Alle Studienteilnehmer wiesen zu Beginn der Studie eine normale rektal-digitale Untersuchung und PSA-Werte unter 4,0 ng/ml auf.

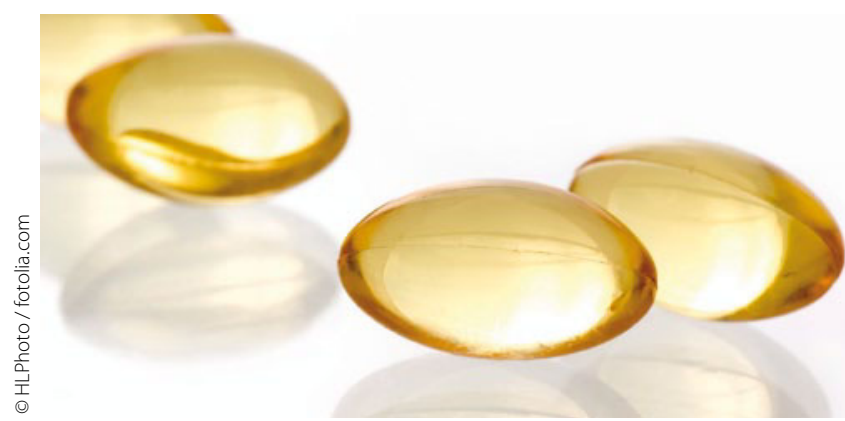

Vom „Zaubermittel“
Vitamin E ist nicht
mehr viel geblieben.

Im Verlauf von 54464 Personenjahren des Follow-up traten in der Placebogruppe 529 Prostatakarzinome auf, in der Vitamin-E-Gruppe beobachtete man 620, in der Selen-Gruppe 575 und in der Kombinationsgruppe 555 Prostatakarzinome. Im Vergleich zu Placebo errechnet sich die Hazard ratio für ein Prostatakarzinom beim Vitamin E mit 1,17 , beim Selen mit 1,09 und bei der Kombination mit 1,05. Die absolute $\mathrm{Zu}$ nahme des Risikos für ein Prostatakarzinom pro 1000 Personenjahre betrug 1,6 für Vitamin E, 0,8 für Selen und 0,4 für die Kombination.

\section{Kommentar}

Eine schlüssige biologische Erklärung für die beobachtete Erhöhung der Rate von Prostatakarzinomen durch Vitamin E ergibt sich aus den Ergebnissen der Studie nicht.
Immerhin sind die Daten insoweit konsistent, als die Kombination von Selen und Vitamin $E$ den ungünstigen Einfluss von Vitamin E auf die Entstehung von Tumoren offensichtlich zum Teil kompensiert. Möglicherweise kann hier die Analyse der noch nicht vorliegenden Konzentrationen von Vitamin D und Selen im Plasma Licht ins Dunkel bringen. Unabhängig von den möglichen Wirkungsmechanismen lässt sich aber heute schon sagen, dass Vitamin E sich nicht nur nicht zur Tumorprophylaxe eignet, sondern möglicherweise sogar zur Erhöhung der Tumorinzidenz beiträgt.

H. S. FÜESSL =

- E. A. Klein et. al.

(Korres.: E.A. Klein, Glickman Urological and Kidney Institute, Cleveland Clinic, Desk Q10-1, 9500 Euclid Ave, Cleveland, OH 44195; kleine@ ccf.org): Vitamin $\mathrm{E}$ and the risk of prostate cancer. JAMA 306 (2011) 14, 1549-1556 